\title{
THE EFFECT OF THREE DIFFERENT POSTURE COMPUTER WORKSTATION ON WORKING PERFORMANCE
}

\author{
Takeshi Sato ${ }^{1}$, Mizuki Nakajima Macky Kato ${ }^{1}$, Ryota Murano ${ }^{2}$ and Hiroshi Morikawa ${ }^{2}$ \\ ${ }^{1}$ Jissen Women's University, 4-1-1, Osakaue Hino Tokyo, Japan \\ ${ }^{2}$ Waseda University, Tokorozawa Saitama, Japan
}

\begin{abstract}
This study aimed to investigate the effect of active workstation designs on sitting, standing and walking during simple typing and pad pointing, and combined tasks for office job. The gradual shift from manufacturing to service industries, combined with technological advanced has resulted in increasing number of people employed in sedentary occupations. It was well known that prolonged sitting was to associate with an increased risk of premature mortality and high levels of sitting cannot be compensated for leisure-time physical activity. Sedentary behavior as sitting computer operation should not be viewed as simply the absence of physical activity or as the extreme lower muscles of lacing physical activity. 13 females performed 20-min office work as typing and searching tasks in each posture, siting, standing desk and standing desk with walking while Oxygen uptake data and HR values was recorded. On the first occasion, a brief description of the test procedures and apparat us was repeated both orally and in writing. They performed on a treadmill to determine their individual oxygen uptake (VO2) and office work like several tasks. Mainly finding showed that walking had significantly higher VO2 than sitting and standing. Moreover, studies have found no effect of Changes in working posture like sitting and standing on computer work performance. Insufficient physical activity is a major threat to health and productivity of office workers. The study confirmed that the difference in energy expenditure of tasks carried out in sitting compared to standing is negligible. Therefore, it is considered that providing exercise habits such as standing and walking while performing office work is an opportunity to eliminate lack of exercise by employees. Rather than sitting all the time, sometimes changing postures and working was considered as one of the health management of employees.
\end{abstract}

\section{KEYWORDS}

Working Posture, Oxygen Uptake, Office Work, Sedentary Worker

\section{INTRODUCTION}

Prolonged sedentary behavior is associated with increased cardiovascular disease risk and decreased energy expenditure due to lacking physical activity. Time spent sedentary is increasing both in the workplace and during everyday life and work. Physical inactivity has long been recognized as detrimental for cardiovascular and metabolic health, however previous studies has remained sedentary behavior as one of the independent health risk factors (Dunstan, et al., 2012). It was well known prolonged sitting is associated with decreased metabolic health, cardiovascular disease and increased mortality. It means that low energy expenditure with sitting is to implicate in the increased prevalence of overweight and obesity, making sedentary behavior an expanding research filed within work-based ICT environment. Therefore, standing computer work is increasingly popular. Standing posture is generally less muscularly demanding than the sitting computer work, neck/shoulder musculature responds evaluated electromyography in each posture during typing tasks. There was not enough data to measure the directory energy expenditure as $\mathrm{VO}_{2}$ during three different working posture. The purpose of this study was to investigate the computer operation tasks and compare the $\mathrm{VO}_{2}$ and heart rate (HR) responses during sitting, standing and walking. 


\section{METHODS}

Thirteen females with no smoking, no cardiovascular and metabolic disease participated in this study. It was performed 20 min pseudo office work test as typing and searching tasks operating computer in three difference posture, siting, standing desk and standing desk with optimum walking recording $\mathrm{VO}_{2}(\mathrm{VO} 2000$, MGC, USA) and HR. It was performed on three different same level computer tasks during three working posture (Figure 1). Data were analyzed during three different posture performance, were compare using ANOVA. When a significant main effect or interaction effect was identified, differences were delineated using a paired Student's t-test. For all comparisons, significance was set at $\mathrm{P}<0.05$. All data are presented as means \pm SD. Statically analyses were performed using SAS University edition.

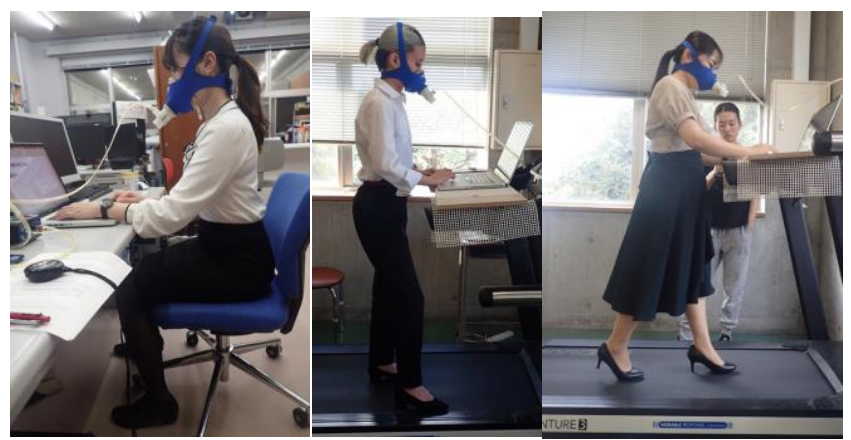

Figure 1. Experimental settings with measurement devices, sitting(left), standing(middle), walking(right)

\section{RESULTS}

There were significantly different between sitting $(7.8 \pm 6.3 \mathrm{ml} / \mathrm{kg} / \mathrm{min})$ and standing $(10.9 \pm 9.4 \mathrm{ml} / \mathrm{kg} / \mathrm{min})$, and walking $(27.6 \pm 17.8 \mathrm{ml} / \mathrm{kg} / \mathrm{min})$ in $\mathrm{VO}_{2}$ (Figure 2). It was normalize performance adjusted standard deviation score as pseudo office work with using personal computer in three conditions. There was no significantly difference performance tasks during 20 min several personal computer operation tests. Moreover, there was no significant difference HR response (Figure 3) for sitting $(76.0 \pm 5.3 \mathrm{beat} / \mathrm{min})$ and standing $(86.2 \pm 9.1 \mathrm{beat} / \mathrm{min})$, and walking $(89.2 \pm 10.4 \mathrm{beat} / \mathrm{min})$.

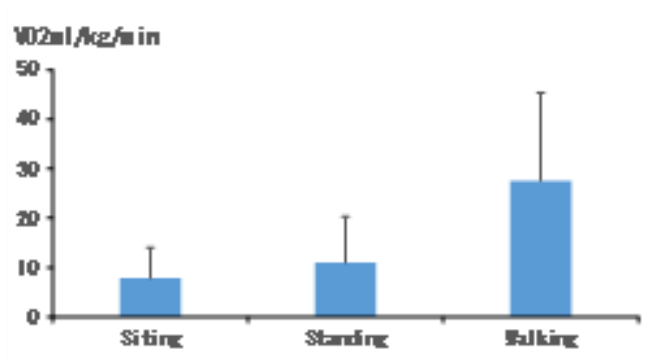

Figure 2. $\mathrm{VO}_{2}$ response in three different working posture 


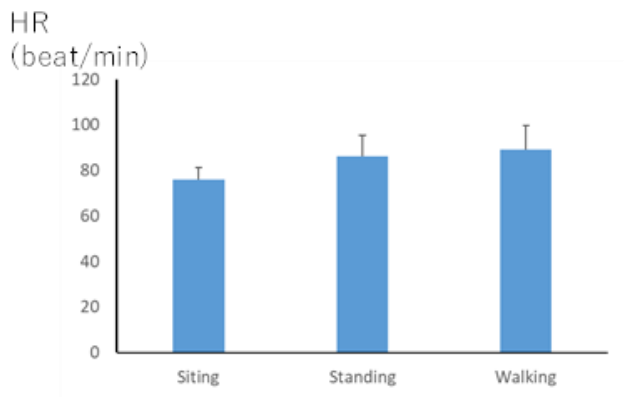

Figure 3. Heart Rate response in three different working posture

\section{DISCUSSION}

This study investigated three different working posture as an alternative intervention choice which could be utilized to interrupt sedentary time. It was important the current definition of sedentary behavior by measuring the energy expenditure of office-based tasks in three different posture. During this experimental task, participants were required to repetitively scroll pages and perform mouse pad operations due to notebook station as well as type texts and simply drawing. Previous study performed the definition of sedentary work behavior using METs as calorimetry (Mansoubi et al., 2015) but included tasks were watching television, just typing, playing PlayStation Portable and Wii that were different from office-work tasks. Therefore, it was performed to walk optimized each other, walking speed was very slowly at around $1.4 \mathrm{~km} / \mathrm{h}$. Then, it was not change in HR responses between standing task and walking task with keeping sedentary physical level. This study found the task of personal computer had a greater effect on VO2 energy expended than the three different posture. This finding would suggest that to increase the energy cost of office activity with personal computer, to perform office-activity on the standing and walking in acceptable conclusion range.

\section{CONCLUSION}

This study demonstrates standing posture are a time efficient method to break up sedentary time, which has the practical potential to be employed in workplaces.

\section{HEADING}

Working posture with walking, standing, sitting.

\section{ACKNOWLEDGEMENT}

We wish to thank the daily help given by Miss. Mana Yoshioka in analyzing the experimental data.

\section{REFERENCES}

Dunstan DW, Howard B, Healy GN, Owen N, 2012. Too much sitting - a health hazard. Diabetes Res Clin Pract, Vol 97, pp. 368-376.

Mansoubi M. Pearson N, Clemes S, Yates T, 2015. Enegy expenditure during common sitting and standing tasks: Examining the 1.5MET definition od sedentary behavior. BMC Public Health, Vol 15, pp.515, doi:10.1186/s12889015-1851-x. 